\title{
Les gorilles du Parc National de Lobéké (Cameroun): interactions avec les populations locales et implications pour la conservation
}

\author{
Samuel Christian TSAKEM ${ }^{1,2, *}$, Martin TCHAMBA ${ }^{3}$ et Robert Bertrand WELADJI ${ }^{4}$ \\ ${ }^{1}$ Ecole Régionale Post-Universitaire d'Aménagement et de Gestion Intégrés des Forêts et Territoires \\ Tropicaux (ERAIFT), Kinshasa, République Démocratique du Congo. \\ ${ }^{2}$ Ecole de Faune de Garoua, BP : 271 Garoua, Cameroun. \\ ${ }^{3}$ Département de Foresterie, Faculté d'Agronomie et des Sciences Agricoles (FASA), Université de Dschang, \\ B.P. 222 Dschang, Cameroun. \\ ${ }^{4}$ Department of Biology, Concordia University, 7141 Sherbrooke Street West, Montreal, \\ QC H4B 1R6, Canada. \\ *Auteur correspondant ; E-mail: ctsakem@yahoo.fr, Tél : (00237) 697692181/672785802
}

\section{RESUME}

La connaissance des interactions entre les populations locales et le gorille est nécessaire pour l'élaboration des stratégies de conservation durable dans une aire protégée. L'insuffisance d'informations de base sur ces interactions a motivé la présente étude autour du Parc National de Lobéké (PNL). Des entretiens et des enquêtes ont été menés auprès des ménages, des gestionnaires de parc et des associations villageoises dans quatre sites autour du parc. Le résultat des enquêtes indique que le gorille est une source importante de revenus à travers le tourisme, les emplois locaux et des réalisations des opérateurs économiques. Cependant, ces retombées sont disparates selon les sites et restent d'ailleurs négligeables auprès des populations. D'autre part, le gorille génère les conflits, puisqu'il détruit les champs des riverains et est braconné par les populations. Les pertes en cultures sont rapportées par plus de 59\% des ménages selon le site et sur près de $30 \%$ des superficies cultivées. Ainsi, les stratégies de redistribution des retombées et de résolution des conflits doivent tenir compte des spécificités de chaque site autour du parc. Il est recommandé de sensibiliser les populations à installer leur champ dans un périmètre où elles maîtrisent le contrôle.

(c) 2015 International Formulae Group. All rights reserved.

Mots clés: Gorille, Tri-National de la Sangha, conflit Homme-gorille, gouvernance.

\section{INTRODUCTION}

La croissance démographique et la rapide conversion des forêts en espace agropastoral ont un impact négatif sur l'habitat de la faune (Hockings et Sousa, 2011 ; De Wasseige et al., 2012). C'est ainsi que plus de $90 \%$ de l'habitat des grands singes africains ont subi des impacts de suite des activités anthropiques (Nelleman et Newton,
2002). Dès lors, les populations locales et la faune sauvage partagent, et de façon conflictuelle, les mêmes espaces et ressources naturelles vitales (Newmark et al., 2004; Hockings, 2009; Aharikundira et Tweheyo, 2011). Les interactions entre la faune sauvage et les communautés riveraines des aires protégées (AP) ont déjà fait l'objet de plusieurs travaux (Weladji et Tchamba, 2003 ; 
Madden, 2008 ; Marchal et Hill, 2009) y compris des études portant sur les gorilles (Madden, 2006 ; Hockings et Humle, 2009 ; Aharikundira et Tweheyo, 2011 ; Hockings et Sousa, 2011).

Autour du parc national de Lobéké (PNL), les populations locales vivent déjà confinées dans les zones d'exploitation forestière et faunique et dont la création a d'ailleurs accentué des restrictions sur l'extension de leurs activités (MINFOF, 2006; Stokes et al., 2008). Malgré l'importance socio-écologique de ce site, il n $\mathrm{y}$ a pas encore des informations de base sur les interactions Homme-gorille dans la périphérie. La littérature existante traite prioritairement de l'écologie des éléphants, des antilopes et des aspects socio-économiques (Ngono et al., 2008 ; Nzooh-Dongmo, 2009). Pourtant, ces interactions peuvent générer d'importantes retombées écologiques et socio-économiques (Gillingham et Lee, 2003). Les mécanismes de gestion de ces retombées sont encore peu connus, de même que la visibilité de leurs impacts sur les populations autour du PNL (Ngono et al., 2008; Donfack et Ajonia, 2010). Les interactions Homme-gorille engendrent aussi des conflits d'utilisation des ressources naturelles (Madden, 2008; Marchal et Hill, 2009; Aharikundira et Tweheyo, 2011). L'importance des conflits Homme-grands singes a amené le Groupe des Spécialistes des Primates de l'Union Internationale pour la Conservation de la Nature (UICN) à en faire une priorité (Hockings et Humle, 2009). Dans le contexte de ce conflit, la destruction de l'habitat et le braconnage sont de loin les principales menaces sur les populations de gorille (Marchal et Hill, 2009). Les dégâts des gorilles sur les cultures autour du PNL ne sont pas encore évalués (Ngono et al., 2008). La faune sauvage reste une source importante de protéine animale pour plus de $75 \%$ de la population humaine dans le bassin du Congo (De Wasseige et al., 2012) et les primates constituent à eux seuls près de $10 \%$ du butin de chasse autour du PNL (Ngwanye, 2008).
C'est ainsi que les grands singes ont connu une diminution de leurs populations depuis une dizaine d'années dans le PNL, allant de 6360 individus en 2002 à 5598 en 2006 et 2685 en 2009 (Nzooh-Dongmo, 2009), en dépit de leur statut d'espèces intégralement protégées au Cameroun et de leur classement dans la liste rouge de l'UICN.

Le gorille de plaine de l'ouest occupe une place importante dans la vie des peuples de forêt autour du PNL en raison de leur relation historique, sociale et culturelle (MINFOF, 2006 ; Donfack et Ajonia, 2010). La récurrence des conflits Homme-gorille suscite le besoin de comprendre la dynamique des interactions autour de ce parc. Ce constat a motivé la réalisation de la présente étude dont les principaux objectifs visent à1) décrire les interactions Homme-gorille, 2) faire un état des lieux des conflits Homme-gorille et 3) évaluer l'impact de ces conflits sur la conservation et sur les populations locales.

\section{MATERIEL ET METHODES \\ Site de l'étude}

Cette étude a été conduite autour du PNL qui couvre une superficie de 217854 hectares, entre les latitudes Nord de $2^{\circ} 05^{\prime}$ à $2^{\circ} 30^{\prime}$ et les longitudes Est de $15^{\circ} 33^{\prime}$ à $16^{\circ} 11^{\prime}$. Plusieurs Unités Forestières d'Aménagement (UFA) sont définies dans la périphérie de ce parc, en superposition aux Zones d'Intérêt Cynégétiques (ZIC) et aux Zones d'Intérêt Cynégétiques à Gestion Communautaire (ZICGC) (Figure 1). Le PNL fait partie du paysage du Tri-national de la Sangha (TNS) avec les parcs nationaux de Dzanga-sangha (République Centrafricaine) et NouabaléNdoki (République du Congo). Les populations riveraines sont issues de deux (02) ethnies dominantes à savoir les Bagando et les Baka. L'agriculture, le petit commerce et la chasse sont les principales activités de ces populations (MINFOF, 2006). Cette zone abrite une flore riche d'environ 764 espèces végétales appartenant à 102 familles et près de 45 espèces de grands et moyens mammifères (Nzooh-Dongmo, 2009). 


\section{Collecte des données}

Deux types de données ont été collectés dans le cadre de cette recherche : (a) des observations directes sur le terrain basées sur l'approche d'Aharikundira et Tweheyo (2011) et (b) une combinaison d'entretiens avec les acteurs de la conservation et d'enquêtes auprès des communautés riveraines du PNL adaptée de Gillingham et Lee (2003) et de Marchal et Hill (2009). Ces investigations ont été menées dans les neufs villages riverains du parc entre les mois de juin et août 2012. Ces villages ont été regroupés en quatre sites en fonction des critères pouvant avoir une influence sur la conservation du parc notamment: l'accessibilité, la présence des infrastructures sociales, la situation géographique, la présence des usines de transformation du bois et la proximité au parc. Les villages situés à l'ouest du parc (Mambélé et Koumela) constituent le Site 1, les villages situés au sudouest (Yenga, Dioula, Mbateka et MbangoyéNguilili) forment le Site 2, les villages situés au sud (Kika et Zega-PK14) forment le Site 3 tandis que le village Libongositué au Nord forme le Site 4.Des entretiens ont eu lieu avec les gestionnaires d'aires protégées (06),les représentants des organismes de conservation (03), les autorités traditionnelles (09)et les associations de développement (08). Le ménage a constitué l'unité d'enquête auprès des populations locales et un questionnaire a été administré à chaque chef de ménage. Cependant, l'idéal était de regrouper lorsque cela était possible, le maximum des membres d'un ménage pour le recoupement des informations fournies. Quatre-vingt-quinze (95) ménages ont été choisis de manière aléatoire et enquêtés, soit vingt-trois (23) dans le site 1 , quarante-quatre (44) dans le site 2 , quinze (15) dans le site 3 et treize (13) dans le site 4 .

\section{Analyse des données}

Le niveau des dégâts sur les cultures pour chaque ménage a été estimé par la proportion des champs dévastés par les gorilles par rapport à la superficie totale du champ. Ainsi trois catégories ont été créées en se référant à Weladji et Tchamba (2003) : faible (< à 25\%), moyen (25-50\%) et élevé (> $50 \%)$. Une évaluation des pertes financières a été faite à partir des prix des denrées affectées sur le marché local au moment de l'étude.

Les données des ménages ont été regroupées par site. Elles ont enfin été résumées et croisées pour les analyses statistiques afin de déterminer si certains paramètres (ethnie, sexe, éducation, lieu de résidence, site) pouvaient influencer la perception des populations sur la conservation, la gouvernance locale, les dégâts sur les cultures et les pertes financières. Pour ce faire, l'ANOVA a été utilisée et le niveau de signification de 5\% a été adopté. Les analyses statistiques ont été effectuées avec le logiciel SPSS 15.0.

\section{RESULTATS}

\section{Contribution des gorilles au développement local}

Il ressort des entretiens avec les acteurs que les avantages liés à la conservation des gorilles sont constitués des dons ponctuels des touristes aux villageois, des divers appuis des bailleurs et organismes de conservation, des revenus de l'écotourisme, des emplois proposés aux riverains ainsi que certaines réalisations des opérateurs économiques du secteur forestier. L'analyse de ces entretiens indique que le gorille et l'éléphant constituent l'attraction principale des touristes dans le parc. Le PNL reçoit en moyen 100 touristes par an qui sont en plus impressionnés par les sites particuliers à l'instar de la base de Djembé, le Lac Lobéké, les clairières forestières et la culture Baka. Dans la pratique, près de $50 \%$ des ménages estiment que leur village ne bénéficie pas de l'arrivée des touristes. Cette proportion varie selon les sites (ANOVA : $\mathrm{F}_{1,3}=12,2, \mathrm{P}=0,001$ ). Dans le site 1 , environ $65 \%$ des ménages estiment que le tourisme constitue le moteur de leur 
développement alors que dans les autres sites, plus de $60 \%$ de la population considèrent que leur village ne bénéficie pas des activités touristiques (Figure 2).La notion d'importance $\mathrm{du}$ tourisme au développement est perçue différemment entre les sites (ANOVA : $\mathrm{F}_{1,3}=9,58, \quad \mathrm{P}=0,002$ )et l'ethnie (ANOVA : $\left.\mathrm{F}_{1,3}=8,25, \mathrm{P}=0,004\right)$.

Chaque année, le Comité de valorisation des ressources fauniques (COVAREF) bénéficie de l'administration forestière de près de 30 millions de francs CFA (1FCFA $\approx 500$ \$US) pour réaliser les infrastructures dans les villages riverains. Ce montant a permis la construction de 2 salles de classe dans les villages riverains, la prise en charge de quelques enseignants vacataires dans les écoles primaires et l'aménagement des hangars dans les chefferies. Cependant, de l'avis de $63 \%$ de la population, ces réalisations restent insignifiantes pour déclencher une réelle dynamique de développement local. Vingt-huit pourcent de ménages jugent ces retombées moyennes tandis que 9\% soutiennent qu'elles sont importantes. Le manque de transparence est ainsi décrié (Figure 3), car 68\% des ménages jugent cette gestion non transparente, ceci indépendamment du niveau d'éducation (ANOVA : $\quad \mathrm{F}_{1,3}=0,87, \mathrm{P}=0,7$ ), $\mathrm{du}$ groupe ethnique (ANOVA : $\mathrm{F}_{1,3}=3,84, \mathrm{P}=0,012$ ) et des sites (ANOVA : $\mathrm{F}_{1,3}=1,91, \mathrm{P}=0,13$ ).

\section{Conflits Homme-gorille}

Les enquêtes ont permis d'identifier les sources de conflits dans la périphérie du PNL. Il s'agit de la concurrence alimentaire $(81 \%$ de ménage), de la demande en gibier $(65 \%)$, la forte croissance démographique qui induit la conversion de l'habitat de la faune en espace agricole (66\%), l'isolement de certains champs $(58 \%)$, la vengeance des populations contre les gorilles $(40 \%)$ et la pauvreté $(59 \%)$ qui prédispose les populations à une dépendance absolue des ressources de la nature. Les conflits Homme-gorille identifiés autour du PNL sont liés soit au comportement des humains (braconnage des gorilles, destruction de l'habitat et l'exploitation anarchique des produits naturels), soit au comportement des gorilles (destruction des cultures, menaces et attaques des animaux domestiques). Les populations rentrent aussi en rivalité avec les gorilles dans l'usage de la plupart des produits naturels à l'instar de : Irvingia gabonensis (93\% de ménage), Annonidium mannii (77\%), Tricocepha accumulata (75\%), Gnetum africanum (47\%), Aframomum sp. (24\%) et Irvingia wombulu (4\%).

Le gorille est de loin la principale espèce responsable des dégâts sur les cultures des populations d'après $94 \%$ de ménages. Les autres espèces, notamment l'éléphant (Loxodonta africana), le cercocèbe (Cercocebus galeritus) et les cercopithèques (Cercopithecus neglectus et pogonias) font des dégâts localisés. Près de $58 \%$ des ménages ont eu régulièrement des dégâts de gorille dans leurs plantations ces deux dernières années. Près de onze (11) types de culture sont cités par les populations comme étant détruits par les gorilles parmi lesquels le bananierplantain (Musa paradisiaca) et le manioc (Manihot esculenta) sont les plus dévastées d'après $96 \%$ et $62 \%$ de ménages respectivement.

Suivant le Tableau 1, seulement $41 \%$ des ménages n'ont pas connu de dégâts de gorilles dans leurs champs ces deux dernières années. L'intensité des dégâts de gorilles sur les cultures ne diffèrent pas entre les sites (ANOVA : $\mathrm{F}_{1,3}=2,296, \mathrm{P}=0,083$ ). Les dégâts sévères sur les cultures sont enregistrés chez $13 \%$ de ménages dans le site 3. Dans chacun des trois premiers sites, près de $14 \%$ des ménages souffrent des dégâts moyens, et dans le site 4 à Libongo, aucune plainte n'a été enregistrée pour ce type de dégât. Le site 1 s'illustre avec les faibles dégâts pour près de $52 \%$ des ménages victimes. D'une manière générale, les dégâts de gorille sont concentrés entre les niveaux faibles et moyens dans les trois premiers sites. Près de $80 \%$ des dégâts 
sont enregistrés en saison sèche. Le niveau des dégâts dans les champs est positivement corrélé (corrélation de Pearson) avec la distance entre le champ et le village $(R=0,26$, $\mathrm{P}=0,011$ ), et cette relation est la même dans tous les sites (ANOVA : $\mathrm{F}_{1,3}=2,30, \mathrm{P}=0,083$ ).

Les pertes sur les cultures concernent près de $59 \%$ déménages et peuvent atteindre $30 \%$ de la superficie des champs. Parmi les ménages victimes, environ $33 \%$ ont subi les pertes financières annuelles inférieures à 50000 FCFA. Les pertes comprises entre 51 et 100 milles FCFA concernent 17\% de ménages (Tableau 2), Les pertes sévères (supérieure à 100000 FCFA) ont été subies par 9\% des ménages, soit $73 \%$ de leur revenu monétaire annuel. Ces pertes ne varient pas significativement d'un site à l'autre (ANOVA : $\mathrm{F}_{1,3}=2,64, \mathrm{P}=0,054$ ).

\section{Comportement des riverains envers la conservation}

Autour du PNL, le gorille est un tabou depuis plusieurs générations pour $26 \%$ des ménages donc 15\% issus de l'ethnie Bodawa. Cette considération devrait réduire la pression sur les populations de gorille, cependant les entretiens menés avec les populations révèlent que cette croyance locale s'est considérablement dégradée avec le temps et la conjoncture. Selon $76 \%$ des ménages, la destruction des cultures, les menaces des humains par les gorilles et les problèmes de gouvernance sont les facteurs qui influençent le comportement des populations vis-à-vis des gorilles et des actions de conservation. C'est ainsi que les communautés riveraines ont développé un sentiment de haine envers la conservation en général et le gorille en particulier. Cette attitude ne diffère pas entre les sites (ANOVA : $F_{1,3}=0,13, P=0,94$ ).

Tableau 1: Niveau de dégâts (en \%) de gorilles sur les cultures dans les villages riverains du PNL.

\begin{tabular}{lccccc}
\hline Niveau de dégât (\%) & $\begin{array}{c}\text { Koumela/ Mambélé } \\
\text { (site 1) }\end{array}$ & $\begin{array}{c}\text { Yenga } \\
\text { (site 2) }\end{array}$ & $\begin{array}{c}\text { Kika } \\
(\text { site 3) }\end{array}$ & $\begin{array}{c}\text { Libongo } \\
\text { (site 4) }\end{array}$ & Total \\
\hline Pas de dégât $(0)$ & 35 & 34 & 33 & 85 & 41 \\
Faible (<25\%) (1) & 52 & 43 & 40 & 8 & 40 \\
Moyen (25-50\%) (2) & 13 & 14 & 13 & 0 & 12 \\
Elevé (>50\%) (3) & 0 & 9 & 13 & 8 & 7 \\
\hline
\end{tabular}

Tableau 2: Pertes annuelles dans les ménages dues aux attaques de gorilles.

\begin{tabular}{lc}
\hline Coût annuel (FCFA) & \% ménages \\
\hline Pas de dégâts & 41.05 \\
$1-50000$ & 32.63 \\
$51000-100000$ & 16.84 \\
$>100000$ & 9.47 \\
\hline
\end{tabular}




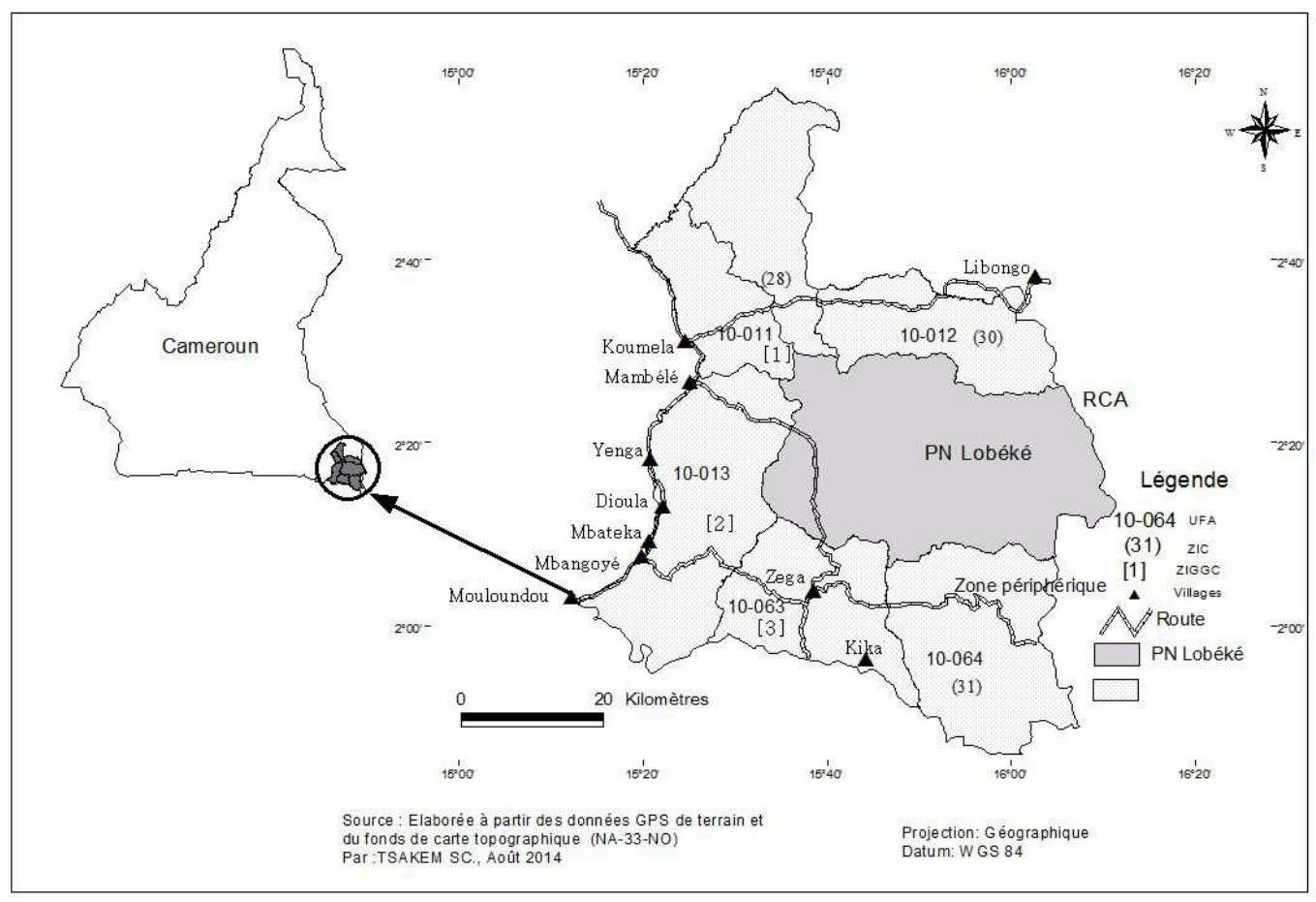

Figure 1: Localisation du Parc national de Lobéké et sa périphérie.

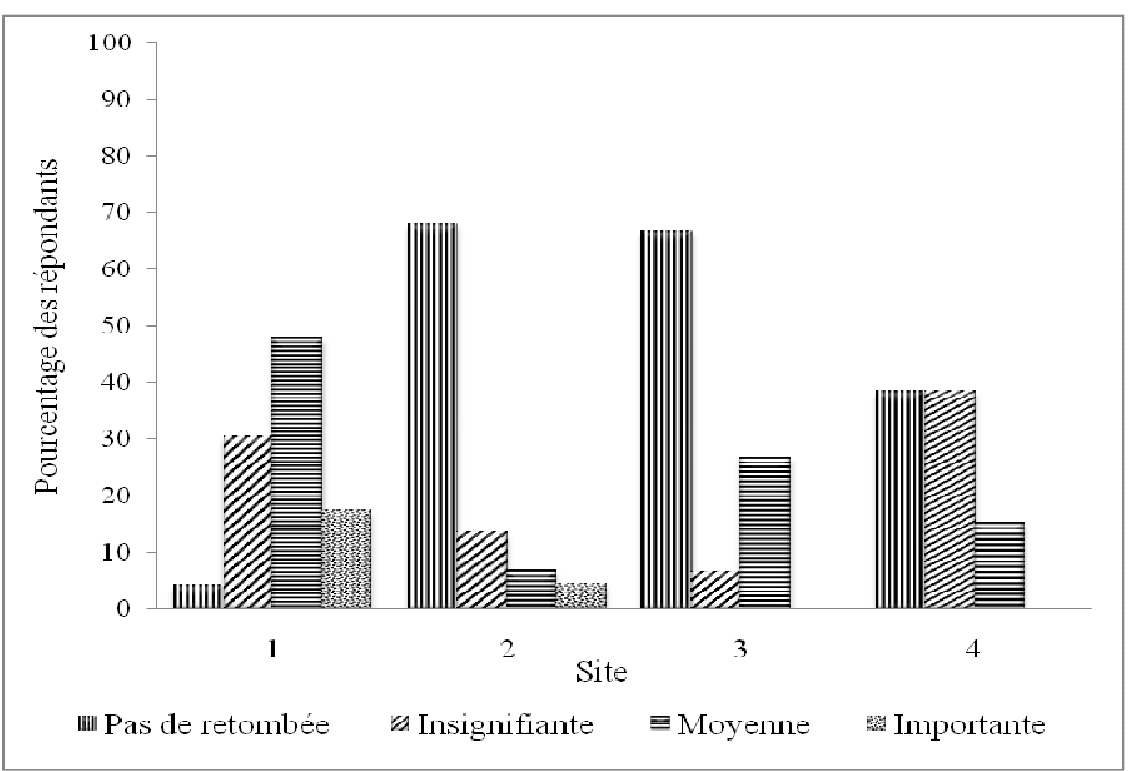

Figure 2: Perception des ménages sur l'importance du tourisme sur le développement local autour du PNL. 


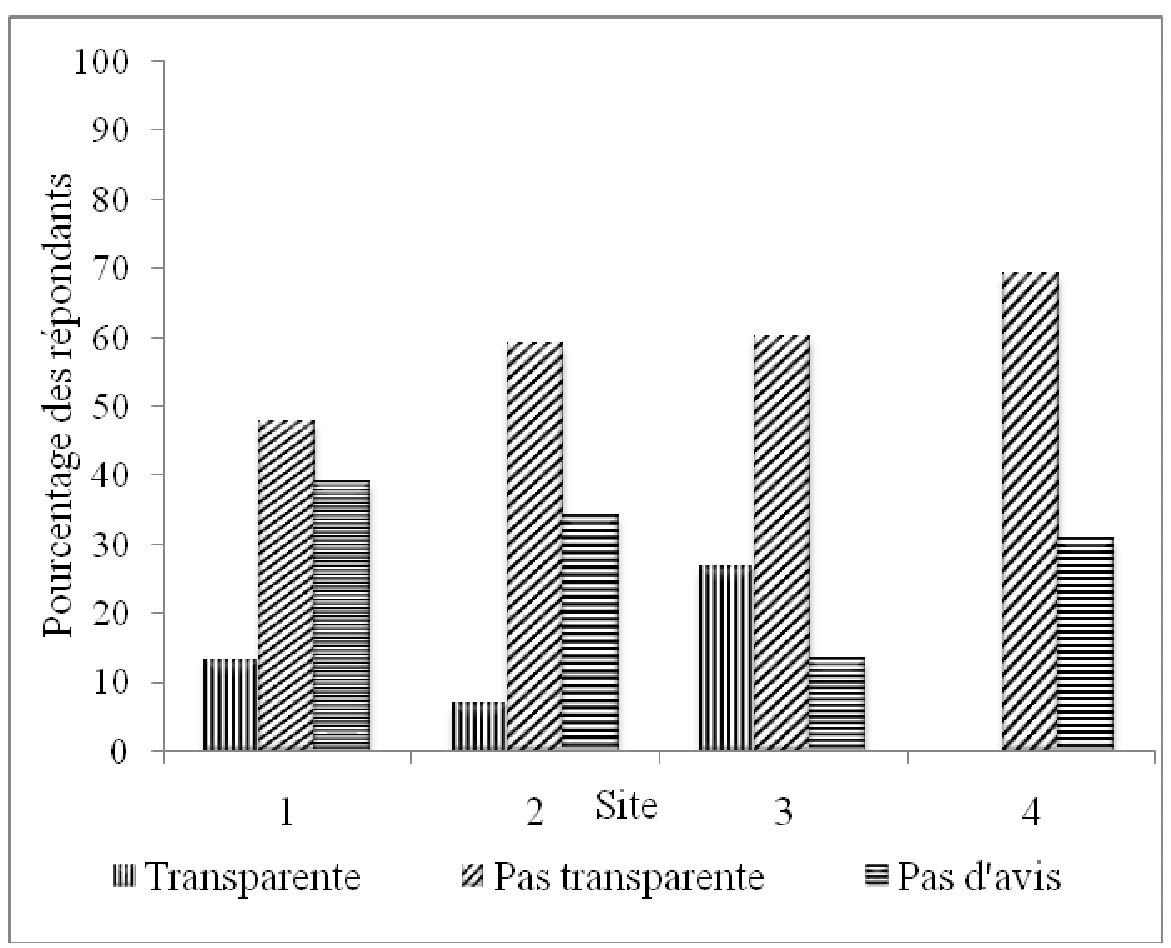

Figure 3: Perception des ménages sur le mode de gestion des retombées de la conservation.

\section{DISCUSSION}

Le tourisme de vision est une meilleure forme de valorisation des espèces sauvages et un outil important de développement pour les communautés locales (Macfie et Williamson, 2010). Autour du PNL, les retombées économiques générées par la conservation ne sont pas encore perceptibles par les populations. Pourtant, l'importance des revenus touristiques sur la conservation a été établie avec succès dans certains sites du Bassin du Congo (Archabald et NaughtonTreves, 2001 ; Sandbrook et Semple, 2006 ; Ancrenaz et al., 2007). La disparité constatée entre les sites dans l'importance du tourisme sur le développement résulte du fait que le site 1 abrite les services de la conservation et les structures d'accueil (camp touristique de Kombo). Il semble qu'autour du PNL, la question de répartition des avantages de la conservation n'est pas bien examinée, ainsi que la priorisation des besoins des populations. Ce qui justifie le fait que les infrastructures sociales réalisées ne sont pas perceptibles auprès de ces communautés.

Certains chercheurs ont noté la confiscation de la gestion des COVAREF par les populations de sexe masculin de l'ethnie majoritaire (Bangando) (Teupa-Lachio et Defo, 2003), ce qui laisserait croire que les aspirations d'autres groupes ne seraient pas suffisamment prises en compte. Cette association est aussi indexée pour la mauvaise gouvernance (Teupa-Lachio et Defo, 2003; Ngono et al., 2008). Une autre cause de l'absence de visibilité des retombées, similaire à celle observée autour d'autres aires protégées du Cameroun (Tsakem, 2009), est le fait que les bénéficiaires font plus de dépenses liées à la consommation d'alcool, à la dot et aux rites traditionnels, ce qui impact faiblement sur l'économie locale.

Comme dans la plupart des aires protégées en Afrique (Madden, 2006; 
Hockings, 2009, Hockings et Humle, 2009), les contacts entre les humains et les gorilles ont souvent lieu dans les espaces protégés, le long des pistes et les lisières forestières et dans les champs de culture. La typologie des conflits Homme-gorille autour du PNL est analogue à celle décrite par (Hockings et Humle, 2009). Le braconnage des gorilles par les populations reste d'actualité dans la zone malgré la réticence des ménages à se prononcer sur le sujet. La consommation de la viande de gibier fait partie de l'habitude alimentaire de tous les ménages enquêtés, reflétant ainsi le contexte sous régional (Hockings et Humle, 2009). Le braconnage commercial des gorilles fait depuis un certain temps, l'objet d'un véritable trafic à la recherche des trophées (mains et crânes) et des jeunes individus comme animaux de compagnies (Hockings et Humle, 2009). L'intensification de la pression sur les gorilles pourrait être la conséquence des avantages de la monétarisation de la faune sauvage. Bien que par le passé les populations aient toujours vécu en parfaite harmonie avec la faune sauvage (Hockings et Humle, 2009 ; Hockings et Sousa, 2011), la question de fond reste leur privation des utilisations traditionnelles des ressources naturelles (Weladji et al., 2003 ; Ngono et al., 2008 ).

Les dommages causés par les gorilles dans les champs ont des impacts sur les populations concernées. Les cultures les plus impliquées représentent les principales sources d'apport glucidique dans l'alimentation des populations riveraines du PNL (Teupa-Lachio et Defo, 2003 ; Stokes et al., 2008) et leur commercialisation permet à ces dernières de satisfaire leurs besoins en produits de premières nécessités. La dévastation de ces cultures fait régulièrement planer l'ombre d'une insécurité alimentaire dans la zone.

La présente étude montre aussi que l'intensité des dégâts de gorille sur les cultures est fonction de la période de l'année et de l'éloignement du champ. Les dégâts sont plus marqués en saison sèche, caractérisée par une rareté des fruits sauvages. En effet, pendant la période de fructification (entre les mois de mai et août), les gorilles sont retranchés en forêt pour s'en approvisionner. Les gorilles de plaine de l'ouest étant essentiellement frugivores (Hockings et Humle, 2009), il existe une relation entre les attaques de gorille et la présence des fruits en forêt (Masi et al., 2009). Les villageois quant à eux s'installent aussi en forêt pour le ramassage des fruits dont le plus sollicité est Irvingia gabonensis. Parmi les produits sauvages conflictuels, si la commercialisation de Annonidium mannii, Gnetum africanum, Aframomum sp, Irvingia wombulu, Tricocepha accumulata se fait à très faible échelle dans les villages, l'Irvingia gabonensis par contre fait l'objet d'un véritable négoce international.

La situation des pertes financières est assez complexe pour les populations quand on sait que dans ces zones reculées, les possibilités d'accumulation des richesses sont très limitées. Alors même que les populations riveraines supportent difficilement la présence des gorilles, ces pertes présagent un futur inquiétant pour toute stratégie de conservation initiée dans la zone. La régularité de ces attaques dans les champs, les pertes financières qui en résultent ainsi que l'absence d'une visibilité des avantages de la conservation des gorilles incitent les populations à une vengeance perpétuelle contre la conservation en général et le gorille en particulier. La principale conséquence est la recrudescence des activités illégales sur les ressources naturelles. Il convient ainsi de s'accorder avec Macfie et Williamson (2010) que l'existence des avantages de la conservation n'entraîne pas toujours la modification du comportement des riverains, ce qui est d'ailleurs contraire à la genèse même de création de ces alternatives. D'autre part, ces avantages seuls ne suffisent pas pour limiter la pression sur les ressources naturelles 
et tout porte à croire que la cause d'une réduction des activités illégales reste un renforcement de l'application de la loi (Macfie et Williamson, 2010) et de la sensibilisation.

Tout comme les ethnies Nalu et Balanta en Guinée Bissau (Hockings et Sousa, 2011), l'ethnie Bodawa autour du PNL a des significations symboliques et religieuses qui offrent une certaine protection traditionnelle au gorille. Malgré les conflits avec les populations, la plupart des scientifiques pensent que le gorille doit continuer à bénéficier d'une attention particulière parce qu'outre son statut de protection, il ressemble à l'Homme et sa disparition s'apparenterait à un effondrement de la vie humaine (Hockings et Sousa, 2011).

\section{Conclusion}

La présente étude montre que la conservation des gorilles dans le PNL apporte des revenus qui ne sont pas encore bien visibles dans les localités riveraines. La présence des aires protégées a occasionné aussi des conflits d'utilisation de l'espace et des ressources. Aussitôt, de nouvelles approches sont nécessaires pour redynamiser les relations entre les communautés locales et le gorille tout en minimisant les pertes subies par les populations. Les stratégies de résolution des conflits doivent tenir compte des spécificités de chaque site. Par exemple, on devrait plutôt développer les techniques visant à limiter les conflits Homme-éléphant dans le site 4 à Libongo où les attaques des pachydermes sont plus récurrentes que celles des gorilles. Même si le regroupement des champs s'avère improbable, il est fortement recommandé de sensibiliser les villageois à installer leur champ dans un périmètre où ils maîtrisent le contrôle. Les recettes de la conservation seules ne suffissent pas pour résoudre tous les problèmes des riverains, d'autres investissements peuvent être faits au bénéfice des villages (construction des infrastructures sociales, renforcement du système de santé etc.). Il est conseillé d'investir sur la valorisation des produits touristiques et d'assurer la perpétuité de ces produits dans le parc et à la périphérie. Les dispositions devront être prises pour augmenter les retombées de la conservation aux communautés (augmentation de la redevance forestière et faunique) et en assurer une meilleure répartition et une gestion transparente de celles-ci. La représentation des peuples Baka dans les instances décisionnelles motivera ces derniers à mettre leur savoir traditionnel au service de la conservation de la zone. Par ailleurs, de gros efforts de sensibilisation et d'information des populations locales doivent être faits, en tenant compte des spécificités de chaque site.

\section{REMERCIEMENTS}

Cette étude a bénéficié de l'appui de certaines organisations à qui nous témoignons notre gratitude. Il s'agit du Programme d'Appui à la Conservation des Ecosystèmes du Bassin du Congo (PACEBCo), de la Wallonie-Bruxelles International (WBI) et de la fondation Rufford. Nous exprimons aussi notre reconnaissance aux responsables du PNL, du bureau WWF de Mambélé et aux populations riveraines de ce parc pour leur hospitalité. Nous pensons bien aux relecteurs anonymes qui ont apporté une contribution significative à l'amélioration de ce travail.

\section{RÉFÉRENCES}

Aharikundira M, Tweheyo M. 2011. HumanWildlife Conflict and its Implication for Conservation around Bwindi Impenetrable National Park. USDA Forest Service Proceedings RMRS.

Ancrenaz M, Dabek L, Oneil S. 2007. The costs of exclusion: recognizing a role for local communities in biodiversity conservation. PLoS Biol., 5: e289.

Archabald K, Naughton-Treves L. 2001. Tourism revenue-sharing around 
national parks in Western Uganda: early efforts to identify and reward local communities. Environ. Conserv., 28: $135-149$.

De Wasseige C, De-Marcken P, Bayol N, Hiol-Hiol F, Mayaux P, Desclée B, Nasi R, Billand A, Defourny P, Eba'aAtyi R. 2012. Les Forêts du Bassin du Congo, Etat des Forêts 2010. Office des Publications de l'Union Européenne : Luxembourg ; 274.

Donfack P, Ajonia G. 2010. Analyse participative des facteurs de déforestation et de la dégradation des forêts dans les paysages transfrontaliers TNS et TRIDOM Cameroun. Rapport d'étude UICN.

Gillingham S, Lee PC. 2003. People and protected Area: a study of local perception of wildlife crop-damage conflict in area bordering the Selous Game Reserve, Tanzania. Oryx., 37: 316-325.

Hockings KJ, Humle T. 2009. Lignes directives pour de meilleures pratiques en matière de prévention et d'atténuation des conflits entre humains et grands singes. Gland, Suisse; Groupe des spécialistes des primates de la CSE/UICN.

Hockings KJ, Sousa C. 2011. HumanChimpanzee Sympatry and Interactions in Cantanhez National Park, GuineaBissau: Current Research and Future Directions. Primates Conserv., 26: 1-9.

Hockings KJ. 2009. Human-chimpanzee competition, coexistence and conflict in Africa. Interact. Stud., 10: 183-205.

Macfie EJ, Williamson EA. 2010. Lignes directrices pour de meilleures pratiques en matière de tourisme de vision des grands singes. Gland, Suisse : Groupe de spécialistes des primates de la CSE/UICN.

Madden FM. 2006. Gorillas in the gardenHuman wildlife conflict at Bwindi
Impenetrable National Park. Policy Matters, 14: 180-190.

Madden FM. 2008. The Growing Conflict Between Humans and Wildlife: Law and Policy as Contributing and Mitigating Factors. J. Int. Wildlife. Law Policy, 11: 189-206.

Marchal V, Hill C. 2009. Primate Cropraiding: A Study of Local Perceptions in Four Villages in North Sumatra, Indonesia. Primates Conserv., 24: 107116.

Masi S, Cipolletta C, Robbins MM. 2009. Western Lowland Gorillas (Gorilla gorilla gorilla) Change Their Activity Patterns in Response to Frugivory. Am. J. Primatol., 71: 91-100.

MINFOF. 2006. Plan d'aménagement du Parc National de Lobéké et de sa zone périphérique, $98 \mathrm{p}$.

Nelleman C, Newton A. 2002. Greats apes, the road ahead. An analysis of great ape habitat, using Globio methodology. United Nation Environnement Programme (UNEP), Nairobi.

Newmark WD, Manyanza DN, Gamassa DM, Sariko HI. 2004. The conflict between wildlife and local people living adjacent to protected areas in Tanzania: human density as a predictor, Conserv. Biol., 8: 249-255.

Ngono L, Ndjounan O, Tegomo-Mbio A, Mokouba M. 2008. Évaluation des COVAREF de l'UTO sud-est Cameroun en 2006 et 2007 : Aspects institutionnels et fonctionnels, partage des retombées et impacts sur la conservation ; participation communautaire et contribution au développement local, rapport d'étude WWF.

Ngwanye VA. 2008. A field report on village subsistence hunting in community hunting zones within the periphery of the Lobéké National Park. WWF CCPO Technical report, $25 \mathrm{p}$ 
Nzooh-DongmoZL, 2009.Summary of trend of large mammal population and spatial distribution dynamics in Lobeke national between 2002-20062009.WWF-CPO report.

Sandbrook CG, Semple S. 2006. The rules and the reality of mountain gorilla (Gorilla beringeiberingei) tracking: how close do tourists get? Oryx, 40: 428-433.

Stokes E, Hoefsloot H, Usongo L, Heinze M. 2008. Affectation des terres dans le complexe d'aires protégées tri-national de la sangha (TNS). Etat d'occupation actuelle des terres et orientations générales d'aménagement.

Teupa-Lachio B, Defo L. 2003. Environnement socio-économique de Mambélé, Yenga, Dioula et Mbateka (Arrondissement de Moloundou). Rapport d'étude pour le WWF.
Tsakem SC. 2009. Analyse de la conservation participative: cas de la congestion autour du parc national de la Bénoué, Nord-Cameroun. Mémoire Master IHEID, Génève, $82 \mathrm{P}$.

Usongo L, Curran B. 1996. Le commerce de la viande de chasse au sud-est Cameroun dans la région trinational. Afr. Primates, 2: 2-5.

Weladji RB, Stein R, Moe ET, Vedeld P. 2003. Stakeholder attitudes towards wildlife policy and the Bénoué Wildlife Conservation Area, North Cameroon. Environ. Conserv., 30: 334-343.

Weladji RB, Tchamba NM. 2003. Conflict between people and protected areas within the Benoue Wildlife Conservation Area, North Cameroon. Oryx, 37: 72-79. 